\title{
Designing Mesoporous Silica Nanoparticles to Overcome Biological Barriers by Incorporating Targeting and Endosomal Escape
}

\author{
Miguel Gisbert-Garzarán, Daniel Lozano, Kotaro Matsumoto, Aoi Komatsu, Miguel Manzano, \\ Fuyuhiko Tamanoi,* and María Vallet-Regí*
}

Cite This: https://dx.doi.org/10.1021/acsami.0c21507

Read Online

ACCESS |

山ll Metrics \& More

回国 Article Recommendations

S1 Supporting Information

ABSTRACT: The several biological barriers that nanoparticles might encounter when administered to a patient constitute the major bottleneck of nanoparticlemediated tumor drug delivery, preventing their successful translation into the clinic and reducing their therapeutic profile. In this work, mesoporous silica nanoparticles have been employed as a platform to engineer a versatile nanomedicine able to address such barriers, achieving (a) excessive premature drug release control, (b) accumulation in tumor tissues, (c) selective internalization in tumoral cells, and (d) endosomal escape. The nanoparticles have been decorated with a self-immolative redox-responsive linker to prevent excessive premature release, to which a versatile and polyvalent peptide that is able to recognize tumoral cells and induce the delivery of the nanoparticles to the cytoplasm via endosomal escape has been grafted. The excellent biological performance of the carrier has been demonstrated using $2 \mathrm{D}$ and $3 \mathrm{D}$ in vitro cell cultures and a tumor-bearing chicken embryo model, demonstrating in all cases

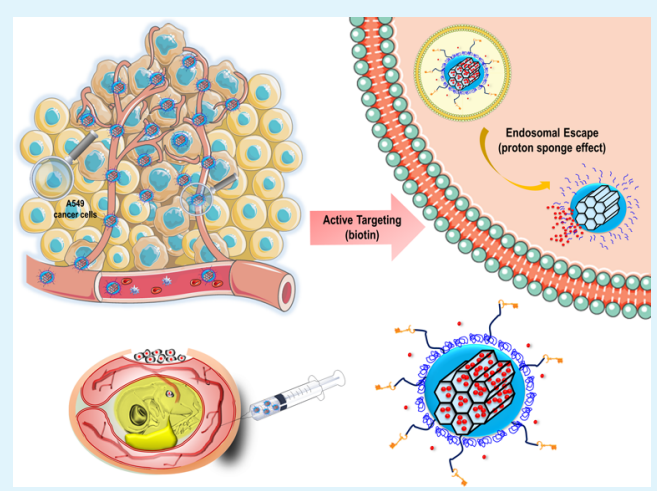
high biocompatibility and cytotoxic effect, efficient endosomal escape and tumor penetration, and accumulation in tumors grown on the chorioallantoic membrane of chicken embryos.

KEYWORDS: mesoporous silica nanoparticles, stimuli-responsive, drug delivery, redox-responsive, self-immolative, targeting, endosomal escape, chicken embryo model

\section{INTRODUCTION}

According to World Health Organization reports, cancer is currently a leading cause of death worldwide, with almost 10 million cancer deaths in $2018 .^{1}$ The number of new cases is expected to reach 25 million over the next two decades. ${ }^{2}$ Among current cancer treatments, chemotherapy lacks tumor tissue selectivity, which leads to nonspecific drug distribution and subsequent toxicity to the patient. In this sense, nanoparticles have emerged as a compelling weapon to encapsulate cytotoxics and deliver them to cancerous cells, reducing those potential side effects. ${ }^{3-5}$ Among all the available nanocarriers, mesoporous silica nanoparticles (MSNs) have drawn attention owing to their exquisite properties, including a network of hollow cavities with the subsequent large surface areas (ca. $1000 \mathrm{~m}^{2} / \mathrm{g}$ ) and pore volumes (ca. $1 \mathrm{~cm}^{3} / \mathrm{g}$ ), tunable pore size and morphologies, facile surface modification, and biocompatibility. ${ }^{6-10}$ Their open porous structure suggests that the cargo molecules could easily diffuse out of the pores before reaching their target cells. This premature release can be diminished using stimuliresponsive gatekeepers. Those are structures that are able to open and close the pore gates in response to certain stimuli (internal or external). ${ }^{11-14}$ That responsive behavior can be accomplished using different types of gatekeepers, such as those based on self-immolative chemistry. ${ }^{15-17}$ These structures degrade in a domino-like fashion when a triggering moiety is removed from the backbone upon application of a very specific stimulus. ${ }^{18,19}$ In this sense, glutathione is overexpressed within the cytoplasm of cancer cells and can be employed to initiate the self-immolation of different redoxresponsive structures. $^{20-25}$

Although research on nanoparticles for drug delivery in cancer has been thoroughly investigated in the laboratory, few of them have reached the bed side. In fact, and despite all benefits that nanoparticles might offer, they have to face several biological barriers that might prevent their effective use for tumor drug delivery. Examples of these barriers include (1) lack of tumor tissue selectivity, (2) lack of selective cancer cell recognition, or (3) endolysosomal sequestration, among others. $^{26,27}$

Received: December 3, 2020

Accepted: February 3, 2021 
Scheme 1. (A) Schematic Representation of the Produced Material in This Work; ${ }^{a}$ (B) Schematic Representation of the Synthesis of the Redox-Responsive Self-Immolative Linker; ${ }^{b}$ (C) Polyvalent Molecule Produced in This Work (HAVAB); ${ }^{c}$ (D) Schematic Representation of the Self-Immolative Degradation of the Linker; ${ }^{d}$
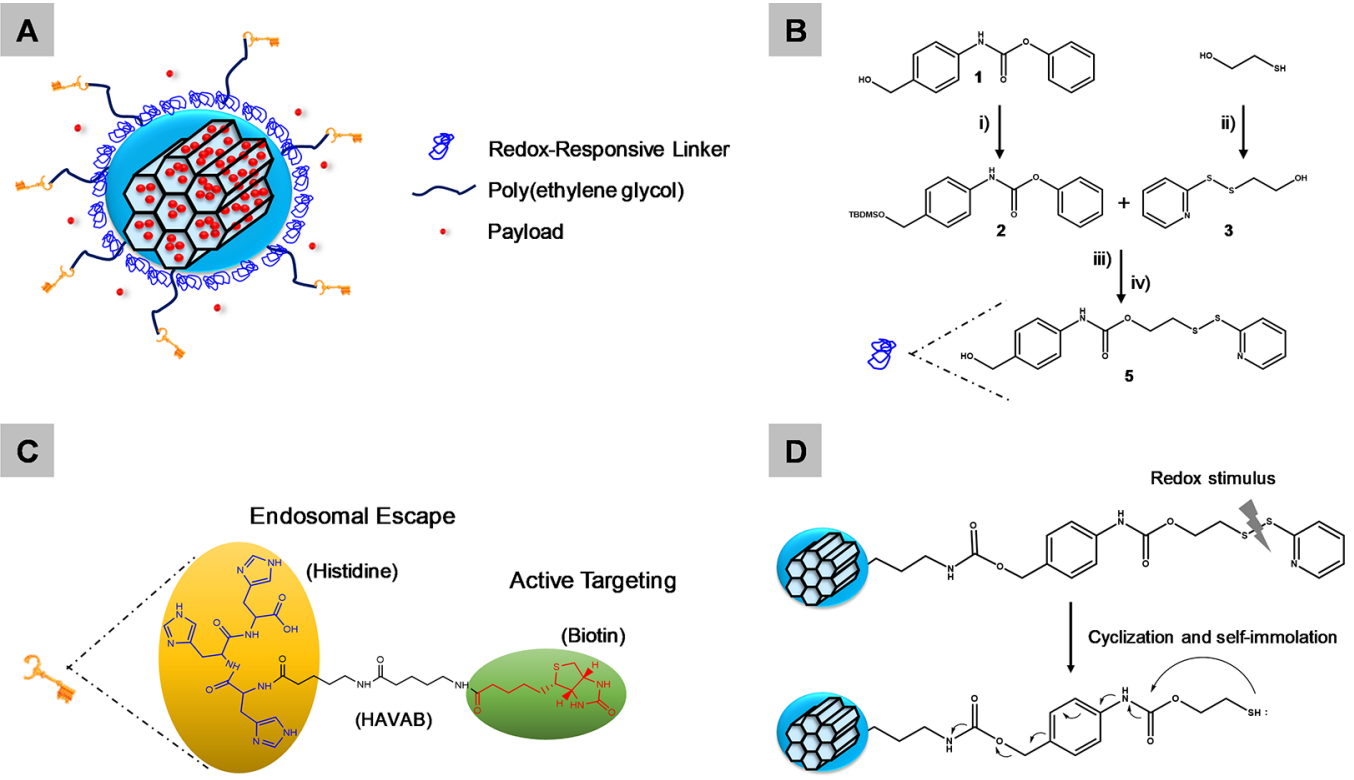

\begin{abstract}
${ }^{a}$ MSNs were functionalized with a silylated redox-responsive linker and subsequently modified with SH-poly(ethylene glycol)-NH $\mathrm{H}_{2}$ ( $\mathrm{PEG}$ ) to finally graft a multifunctional molecule (denoted as HAVAB). The PEG chains were attached to the linker via thiol exchange, forming a disulfide bond. HAVAB was coupled to the PEG using carbodiimide chemistry, leading to the formation of an amide bond. ${ }^{b}$ Reaction steps: (i) imidazole, tert-butyldimethylsilyl chloride, dichloromethane, room temperature, overnight; 2,2'-Dipyridildisulfide, acetic acid, methanol, room temperature, overnight; (iii) DBTL, toluene, reflux, 4 h; (iv) pTsOH, dichloromethane:methanol (1:1), reflux, overnight. For more details, please see Scheme S2. ${ }^{c}$ The part in the right (green) is able to recognize cancer cells and can be tuned on-demand, increasing the versatility of the carrier. The part in the left (yellow) is able to promote endosomal escape via proton sponge effect thanks to the histidine residues. ${ }^{d}$ Upon application of a redox stimulus, the disulfide bond is cleaved, producing an electron pair that attacks the carbamate through cyclization, triggering the 1,6-self-immolation.
\end{abstract}

In this sense, it is well-known that the presence of fenestrations in the tumor blood vessels and the poor lymphatic drainage in the tissue promotes the passive accumulation of nanomedicines in the tumors. In consequence, the so-called enhanced permeability and retention (EPR) effect would provide an effective approximation to tackle the lack of tumor tissue selectivity. ${ }^{28}$ In addition, the overexpression of specific membrane receptors of tumoral cells enables their selective recognition by modifying the nanoparticles surface with molecules showing high affinity for those receptors. In this manner, nanoparticles would be selectively internalized, thereby addressing the lack of selectivity toward tumoral cells. ${ }^{29,30}$ Following internalization of nanocarriers, they might be sequestered within the acidic endolysosomes, which might lead to cargo degradation and reduced therapeutic effect. ${ }^{26}$ In this sense, these vesicles might be disrupted by modifying the particles with species with buffering capacity. These moieties would induce the influx of protons and chloride ions along with water molecules (proton sponge effect), causing the vesicle to swell and expand with the subsequent membrane rupture, thus avoiding the endolysosomal entrapment. ${ }^{31}$

In this work, we have developed a redox-responsive mesoporous silica nanocarrier that is able to slow down premature drug release and showing enhanced tumor accumulation, cancer cell targeting, and endosomal escape capabilities (Scheme 1).

The nanocarriers were endowed with stimuli-responsiveness using a redox-responsive self-immolative linker. The particles were further modified with a versatile molecule containing biotin and histidine to target cells and induce the endolysosomal escape, respectively. The biological performance of these nanoparticles was extensively studied in vitro (2D and 3D tumoral models) and using a tumor-bearing chicken embryo model, demonstrating good performance regarding tumor accumulation, cytotoxicity, and escape from the endolysosomes.

To the best of our knowledge, this is the first time that the active recognition of particles and their subsequent endosomal escape have been demonstrated in 2D and 3D tumor models using such a simple molecule, paving the way for future nanomedicines that might improve the efficiency of cancer treatments.

\section{RESULTS AND DISCUSSION}

Targeted Redox-Responsive Mesoporous Silica Nanoparticles. The synthesis of MSNs was carried out using a modified Stöber method, employing TEOS as silica source and $\mathrm{CTAB}$ as structure directing agent, to obtain colloidally stable and homogeneous spherical nanoparticles presenting hexagonally ordered cylindrical mesopores (see Supporting Information). Then, the particles were endowed with stimuli-responsiveness behavior by grafting a redoxresponsive self-immolative molecule acting as linker and gatekeeper. This linker would be cleaved thanks to the difference in the concentration of glutathione in cancer cells (2-10 $\mathrm{mM}$ in the cytoplasm vs $2-20 \mu \mathrm{M}$ in the extracellular fluids). ${ }^{32,33}$ A comprehensible representation of the synthesis of the linker is depicted in Scheme 1B. As shown there, the redox-responsive self-immolative linker $\mathbf{5}$ was synthesized from compounds $\mathbf{2}$ and 3 . Compound $\mathbf{2}$ was a protected analogue of 

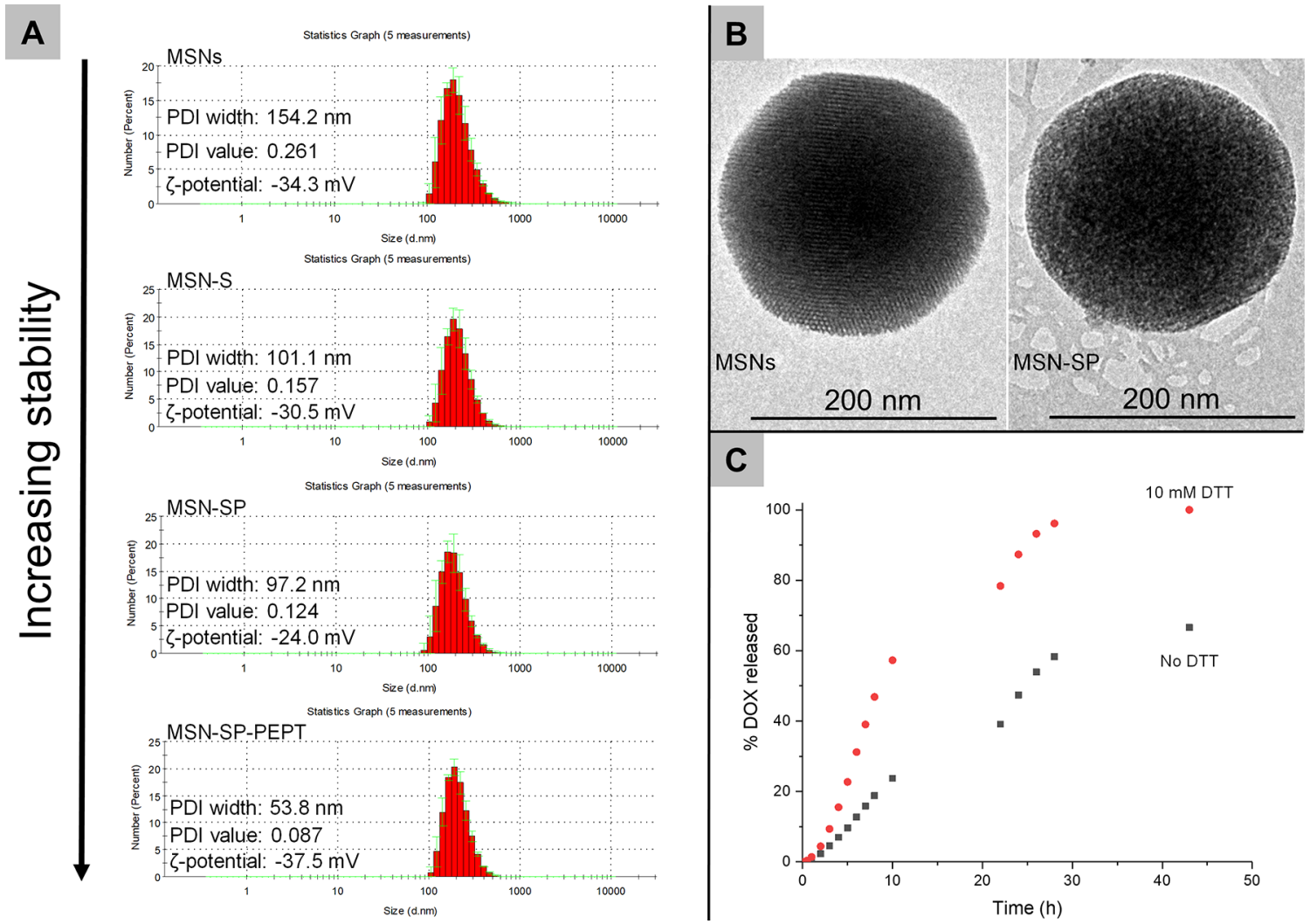

Figure 1. Representative characterization of the mesoporous silica nanoparticles developed in this work. (A) DLS measurements of MSNs, MSN-S, MSN-SP, and MSN-SP-PEPT. The dispersibility of the material increased after each of the functionalization steps, as confirmed by the decreasing values of the PDI width. This value is obtained from the width of the distribution at half its maximum height value. Hence, the lower the value, the more monodispersed the sample and smaller the amount of aggregates in the sample. (B) TEM micrographs of MSNs vs MSN-SP. The samples were stained with phosphotungstic acid, and the blurrier surface of MSN-SP indicated that the surface of the nanoparticles contained organic matter. (C) Release experiment of MSN-SP. The experiment was carried out using Transwells, at $37{ }^{\circ} \mathrm{C}$ and under orbital stirring. DOX was employed as cargo. One group was treated with DTT in PBS $1 \times$ as redox stimulus, while the other received only PBS $1 \times$. The group treated with $10 \mathrm{mM}$ DTT PBS $1 \times$ showed a 2 -fold DOX release compared with the control group (PBS $1 \times$ ) at $24 \mathrm{~h}$ and released additional ca. $40 \%$ after $48 \mathrm{~h}$, compared with the control group that did not receive any stimuli.

a standard self-immolative monomer, ${ }^{34}$ which was synthesized from commercially available 4-aminobenzyl alcohol. Compound 3 was synthesized by reacting 2 -mercaptoethanol with 2,2 '-dipyridildisulfide to introduce the disulfide bond functionality responsive to redox species. Afterward, compounds 2 and 3 were reacted in the presence of a tin catalyst, and the benzyl alcohol was finally deprotected under acidic conditions to accomplish self-immolative compound $\mathbf{5}$.

The redox-responsive linker was then grafted to the particles via silylation and subsequent condensation on the particles surface to yield MSN-S. The grafting process was optimized in terms of (1) the most adequate solvent for the silylation, (2) the most convenient mixture of solvents for the grafting to the nanoparticles, and (3) the best MSNs:Linker ratio. Overall, the silylation was found to be most effective in DCM, as demonstrated by the changes in the chemical environment of the benzylic alcohol of $\mathbf{5}$ (reduction of signal 1 along with appearance of signal 4) in the ${ }^{1} \mathrm{H}$ NMR spectrum (Figure $\mathrm{S} 10$ ). Then, a mixture of $\mathrm{DCM} / \mathrm{AcN}$ was observed to provide the best results for the grafting, as demonstrated by the higher weight loss observed in the TG analysis (Figure S11). Finally, the higher amount of additional organic matter observed by TG analysis confirmed $1: 7$ as the best ratio for the functionalization (Figure S13).
MSN-S were PEGylated (MSN-SP) via thiol exchange with the redox-responsive linker. In this regard, increased colloidal stability was observed as the different functionalization steps were carried out (Figure 1A), as demonstrated by the decreasing values of the polydispersity index (PDI) width. The successful outcome of the different reaction steps was confirmed by the weight loss observed (ca. 24\% for MSN-S and ca. $26.5 \%$ for MSN-SP) in the TG analysis (Figure S14) as well as the changes in the FTIR spectrum, namely, the bands from the carbamate group of $\mathbf{5}$ and the $\mathrm{C}-\mathrm{H}$ groups of the PEG chains) in the FTIR spectrum (Figure S15). The success of each functionalization reaction was also confirmed by the variations in the zeta potential, from $-34.3 \mathrm{mV}$ (MSNs) to $-30.5 \mathrm{mV}$ (MSN-S) and $-24.0 \mathrm{mV}$ (MSN-SP). Besides, the presence of organic matter was confirmed through TEM microscopy upon treatment of MSN-SP with phosphotungstic acid (Figure 1B). Additional physicochemical characterization can be found in the Supporting Information.

The redox-responsiveness and subsequent self-immolation of the linker were evaluated using a solution containing dithiothreitol (DTT). A comprehensible representation of the self-immolative process is depicted in Scheme 1D. Briefly, the presence of redox species triggers the cleavage of the disulfide bond of either MSN-S or MSN-SP, generating a free electron pair. Such electron pair undergoes cyclization onto the 
carbamate, consequently initiating the 1,6-self-immolation of the linker. ${ }^{18,35,36}$ Both MSN-S and MSN-SP were incubated with the reducing agent, showing appreciable changes in their $\mathrm{C}, \mathrm{N}, \mathrm{S}$ composition that were ascribed to the self-immolation of the linker (see Supporting Information). Finally, MSN-SP was loaded with doxorubicin (DOX) to evaluate the responsiveness to redox stimuli. The experiment showed that the redox-triggered material released a 2 -fold amount of cargo at $24 \mathrm{~h}$ and ca. $40 \%$ of additional drug release at $48 \mathrm{~h}$, compared with the control group (Figure 1C), confirming the redox-responsiveness of the material and the feasibility of using a self-immolative construct to hamper excessive drug release.

One of the key parts of this work was the design of a multifunctional molecule that is able to mediate the active recognition of the redox-responsive particles by cancer cells and to promote their subsequent endosomal escape into the cytoplasm (Scheme 1C). HAVAB was produced by employing solid-phase peptide synthesis. Then, it was attached to the particles using carbodiimide chemistry between the C-terminal of the peptide and the amino groups of the PEG chain (see Supporting Information), yielding MSN-SP-PEPT. These nanoparticles exhibited the lowest PDI values and, consequently, the highest colloidal stability (Figure 1A). The rationale behind the increasing colloidal stability as the different functionalization steps were carried out relies on different factors. First, MSN-S was produced by attaching a carbamate-containing self-immolative linker, showing a lower PDI width than MSNs. This is in agreement with our previous results, which showed that functionalizing the surface of pristine MSNs or different hydrophobic mesoporous carbon nanoparticles with a carbamate-based self-immolative polymer resulted in increased colloidal stability. ${ }^{15,17}$ Then, MSN-S was functionalized with PEG, displaying lower PDI width than MSN-S. This would be a consequence of the hydration layer generated around the PEGylated nanoparticles, which has been shown to increase the colloidal stability of different mesoporous silica nanoparticles. ${ }^{37,38}$ Finally, the attachment of HAVAB, a readily water-soluble peptidic molecule, produced MSN-SP-PEPT, which showed the lowest PDI width, highlighting its colloidal stability and demonstrating its suitability to be employed as drug delivery system via systemic administration.

2D Biological Evaluation: Cellular Uptake. Because biotin receptors are overexpressed in many tumoral cells, its conjugation to different types of nanoparticles has yielded nanomaterials with application in imaging, sensing, and drug delivery. ${ }^{39}$ Indeed, its conjugation to $\mathrm{MSNs}$ has been demonstrated to improve their internalization in biotinreceptor-positive cells. ${ }^{37,40-42}$ In this sense, the presence of the biotin targeting moiety promoted the preferential accumulation of MSN-SP-PEPT in biotin-receptor-positive cells rather than in healthy cells (see Supporting Information). Then, the effect of HAVAB on the cellular uptake was further evaluated by flow cytometry, using fluorescein-labeled nanoparticles and analyzing the fluorescence intensity inside the cells. To that aim, MSN-SP-PEPT and MSN-S were placed with HeLa cells. Besides, nanoparticles functionalized with biotin but without the amino acids, denoted as MSN-SP-Biotin (MSNs + linker + PEG + Biotin), was also included (Figure 2A). MSN-SP-PEPT displayed the performance, showing greater internalization for all the concentrations studied in two independent experiments ( 75 and $37.5 \mu \mathrm{g} / \mathrm{mL} ; 18$ and 9 $\mu \mathrm{g} / \mathrm{mL})$, highlighting the remarkable influence of HAVAB on
A

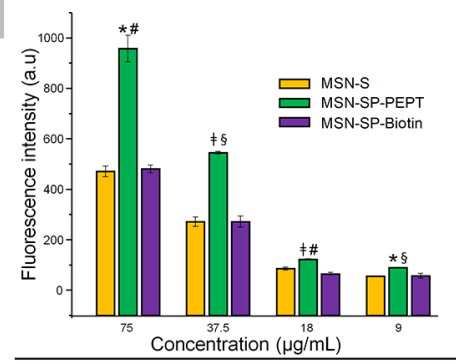

C
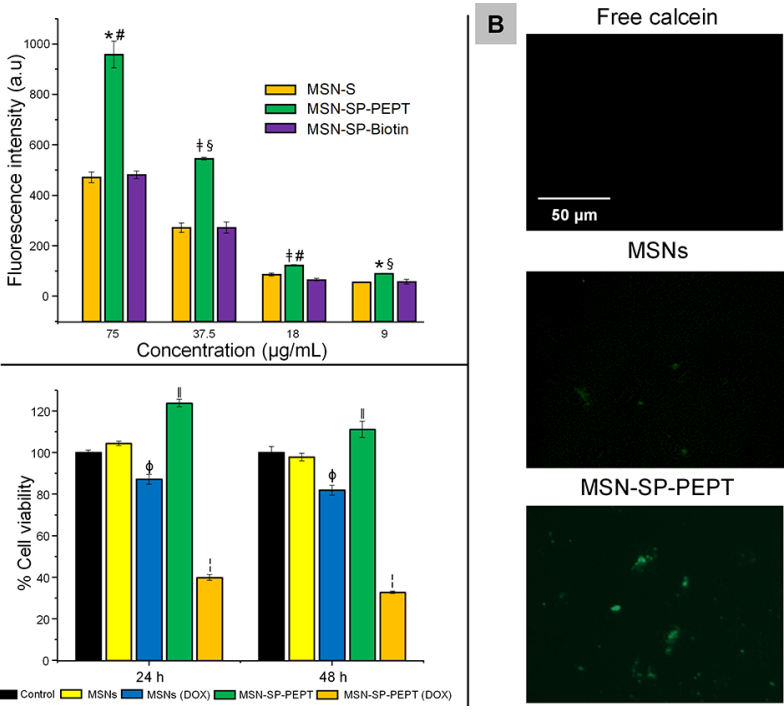

Figure 2. $2 \mathrm{D}$ cellular models. Data are mean \pm standard error of the mean (SEM) ( $n=3$ in all cases). Statistical analysis was carried out using two-tailed student's $t$ test $(p<0.05$ was considered to be significant). (A) Cellular uptake quantified by flow cytometry of fluorescein-labeled MSN-SP-PEPT vs. MSN-S vs. MSN-SP-Biotin in HeLa cells. The highest internalization degree was obtained for MSNSP-PEPT for all the experiments. \# $p<0.05$ vs MSN-SP-Biotin; $\S p<$ 0.05 vs MSN-SP-Biotin; * $p<0.05$ vs MSN-S; $\neq p<0.01$ vs MSN-S. (B) Endosomal escape of free calcein (control), MSNs and MSN-SPPEPT. Green fluorescence was only detected for MSN-SP-PEPT, confirming the endosomal escape. (C) Viability of A549 cells employing drug-free and DOX-loaded MSN-SP-PEPT and MSNs (50 $\mu \mathrm{g} / \mathrm{mL})$. MSN-SP-PEPT was shown to be biocompatible and induced the highest inhibition of the cell viability when loaded with a drug. $\| p<0.01$ vs control; $\phi p<0.01$ vs MSNs and control; ${ }_{\mid} p<0.01$ vs MSN-SP-PEPT, MSNs (DOX) and control.

the cellular uptake. The noticeable difference between MSNSP-Biotin and MSN-SP-PEPT might rely on the effect of $\mathrm{pH}$ on the histidine residues, as they progressively protonate as $\mathrm{pH}$ acidifies. ${ }^{43}$ Then, the remarkable cellular uptake of MSN-SPPEPT might be explained as a consequence of two possible phenomena, which might reinforce the active binding of biotin: (1) the partial protonation would lead to local positive charge effects, and the material would interact more tightly with the cell membrane, which is negatively charged; or (2) it has been demonstrated that peptides or proteins that present histidine residues may lead to membrane fusion upon protonation, ${ }^{44-47}$ which is a phenomenon known to mediate the internalization of some cell-penetrating peptides. ${ }^{48}$

2D Biological Evaluation: Endosomal Escape. Histidine was used owing to the buffering capacity of the imidazole ring at the acid $\mathrm{pH}$ of endosomes, which can lead to the escape of the nanoparticles from the endosomes via proton sponge effect. $^{49-51}$ The endosomal escape capabilities were evaluated loading the particles (MSNs or MSN-SP-PEPT) with calcein (Figure 2B). The calcein method is a standardized approach to evaluate the endosomal escape, owing to its membraneimpermeable nature. ${ }^{52}$ Its fluorescence is self-quenched at high concentrations, such as when it is trapped in the endolysosomes, and it can be detected again when calcein leaves the vesicles, as a consequence of their disruption. ${ }^{53-55}$ As shown in Figure 2B, MSNs loaded with calcein produced low green fluorescence as a result of the nanoparticles being trapped in the endolysosomes. However, cells incubated in the 

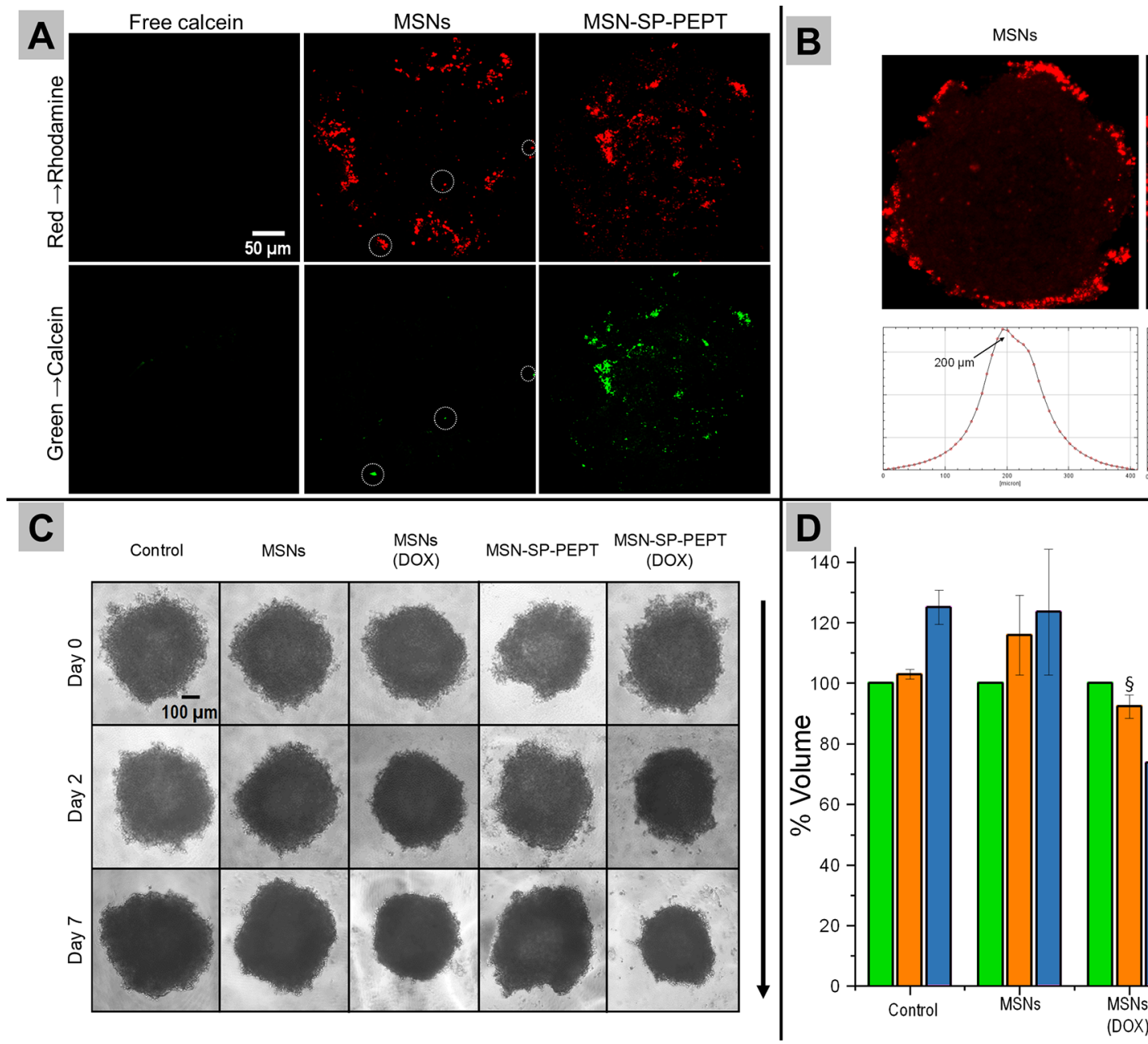

MSN-SP-PEPT
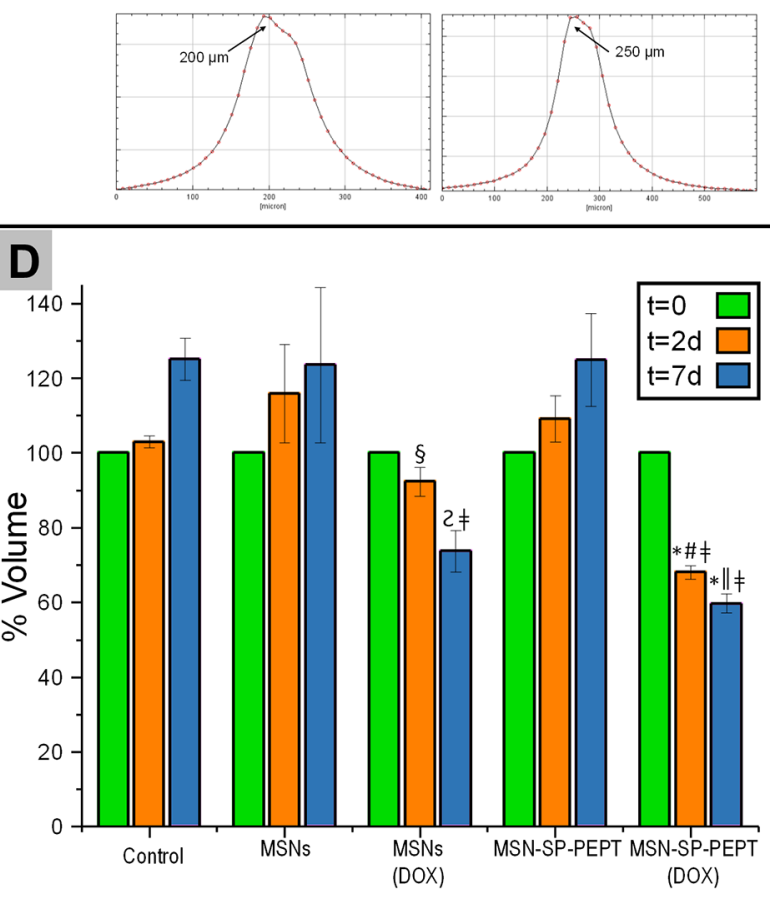

Figure 3. 3D cellular models. Red and green fluorescence indicate rhodamine B-labeled nanoparticles and calcein, respectively. (A) Endosomal escape of free calcein, MSNs and MSN-SP-PEPT. A strong signal was detected for MSN-SP-PEPT, indicating the achievement of endosomal escape. (B) Internalization of MSNs vs. MSN-SP-PEPT using confocal microscopy (top) and depth analysis of MSNs vs. MSN-SP-PEPT employing the software ImageJ (bottom). The nanoparticles bearing HAVAB could reach deeper depth $(250 \mu \mathrm{m}$ vs $200 \mu \mathrm{m})$, demonstrating higher penetration ability. (C) Imaging of spheroids incubated with drug-free and DOX-loaded MSN-SP-PEPT and MSNs (70 $\mu \mathrm{g} / \mathrm{mL})$. The latter group showed the best spheroid inhibition. (D) Viability of A549 spheroids in terms of \% volume employing DOX-loaded and drug-free MSN-SP-PEPT and MSNs $(70 \mu \mathrm{g} / \mathrm{mL}) . \S p<0.05$ vs Control; $\neq p<0.01$ vs Control; $* p<0.01$ vs MSN-SP-PEPT; ? $p<0.05$ vs MSN-SP-PEPT; \# $p<0.01$ vs MSNs (DOX); $\| p<0.05$ vs MSNs (DOX); ${ }_{\mid} p<0.05$ vs MSNs.

presence of MSN-SP-PEPT loaded with calcein displayed strong green fluorescence with a slight diffusion pattern. This was associated with successful escape from the endosomes, ${ }^{56}$ likely through proton sponge effect, ${ }^{57}$ verifying the endosomal escape features of this nanocarrier.

2D Biological Evaluation: Cytotoxicity. The particles (MSN-SP-PEPT or MSN) were loaded with doxorubicin and placed with A549 cells to evaluate their cytotoxicity on tumoral cells. The amount of DOX loaded in the nanoparticles was analyzed by thermogravimetric analysis and was found to be ca. 4\%. As shown in Figure 2C, drug-containing MSN-SP-PEPT significantly inhibited the cell viability (ca. $60 \%$ at $24 \mathrm{~h}$ and ca. $70 \%$ after $48 \mathrm{~h}$ ). The much higher cytotoxic effect of this carrier compared with the control group was ascribed to the enhanced uptake of the particles bearing HAVAB. This effect was observed to be independent of the tumoral cell line employed because MSN-SP-PEPT could also inhibit the viability of HeLa cells (see Supporting Information), confirming the versatility of the produced nanocarrier in this work.
3D Biological Evaluation: Endosomal Escape and Penetration in Tumor Spheroids. The endosomal escape features of the system were also analyzed using tumor spheroids grown from A549 cells (Figure 3A). This in vitro 3D tumor model constitutes a powerful tool to evaluate the biological performance of nanoparticles as it shows several features of in vivo tumors. ${ }^{58}$ Calcein-loaded nanoparticles (MSNs or MSN-SP-PEPT) were used. The results were analogous to those in the 2D tumoral model. Although MSNs remained trapped in the endolysosomes, as demonstrated by the negligible green fluorescence detected, a strong fluorescence was observed for MSN-SP-PEPT, clearly indicating the achievement of the endosomal escape in the $3 \mathrm{D}$ tumor model and confirming the versatility of the nanocarrier.

The distribution of particles in the spheroids was also evaluated. As observed in Figure 3B, MSN-SP-PEPT accumulated much more than bare MSNs in tumoral spheroids, highlighting again the positive effect of HAVAB. The depth reached by the nanoparticles, analyzed employing ImageJ, demonstrated that MSN-SP-PEPT could penetrate 

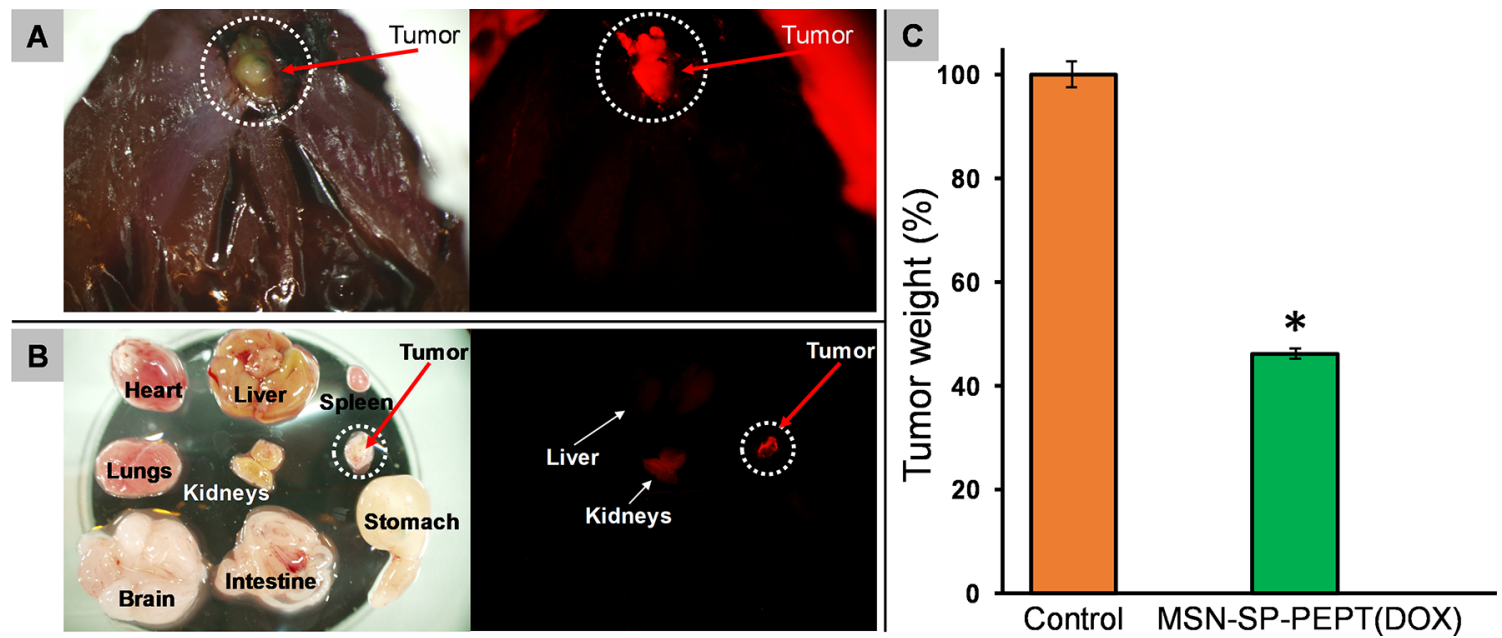

Figure 4. (A) Biodistribution of MSN-SP-PEPT $48 \mathrm{~h}$ after the injection by fluorescence microscopy. Bright field (Left) and red fluorescence (right). Labeled nanoparticles displayed red fluorescence in the tumor, verifying their passive accumulation via EPR effect. (B) Visualization of the organs and tumor from the dissected chicken embryo. Bright field (left) and red fluorescence (right). MSN-SP-PEPT selectively targeted the tumor, showing only minor accumulation in the kidneys and the liver. (C) Tumor growth inhibition by DOX-loaded MSN-SP-PEPT at a concentration of $100 \mu \mathrm{g} / \mathrm{mL}$. In this preliminary study, the nanocarrier showed its capability to lessen the weight of the tumor by ca. $50 \%$ after $72 \mathrm{~h}$. $* p<0.05$ vs. Control. Data are mean \pm SEM $(n=5)$. Statistical analysis was carried out using two-tailed student's $t$ test $(p<0.05$ was considered to be significant).

deeper in the spheroid $(250 \mu \mathrm{m}$ for MSN-SP-PEPT vs $200 \mu \mathrm{m}$ for MSNs). OVCAR8 spheroids were also incubated with such nanoparticles, obtaining analogous results (see Supporting Information). The rationale behind this higher penetration might rely on the endosomal escape. On the one hand, it is known that endolysosomal sequestration reduces the rate of exocytosis of nanoparticles. ${ }^{59,60}$ Besides, Lu et al. demonstrated that nanocarriers penetrate deeper in tumor spheroids via transcytosis if the nanoparticles have undergone exocytosis from the outer cells of the spheroid. ${ }^{61}$ In addition, Cui et al. established the endolysosomal entrapment of nanoparticles as a limiting factor for achieving transcytosis. ${ }^{62}$ In consequence, MSN-SP-PEPT were preferentially internalized owing to the overexpressed biotin receptors to then escape from the endosomes. This would lead to the nanoparticles being exocytosed toward inner areas of the A549 tumor spheroids, to then undergo further cellular uptake through transcytosis, thus improving the final tumor penetration.

3D Biological Evaluation: Cytotoxicity. The particles (MSNs or MSN-SP-PEPT) were loaded with doxorubicin to further evaluate their cytotoxic effect on A549 tumor spheroids. The cytotoxicity was studied by analyzing the spheroid volume at different time points. In this sense, the spheroid images shown in Figure 3C were numerically represented in Figure 3D to facilitate the understanding of the trends observed in Figure 3C. ${ }^{63}$ The spheroids were unaffected by unloaded MSN-SP-PEPT, confirming again the high biocompatibility of the carrier and laying the foundations for future analysis using animals. Moreover, MSN-SP-PEPT loaded with DOX reduced the volume of the spheroids by ca. $30 \%$ after just $48 \mathrm{~h}$ and ca. $40 \%$ after a week, corroborating their capability to penetrate in the tumoral spheroids and deliver cytotoxic compounds to the cancerous cells. Furthermore, drug-loaded MSN-SP-PEPT led to greater cytotoxic effect than drug-loaded MSNs, in agreement with all the previous results. Analogous results were obtained when a lower particles concentration was employed (see Supporting Information).
Tumor-Bearing Chicken Embryo Model. The chicken embryo model allows the growth of human-like tumors, providing valuable information about the biodistribution and cytotoxic effect of nanoparticles. ${ }^{64,65}$ The tumors were grown within 3-4 days by placing the tumoral cells in a Teflon ring on the chorioallantoic membrane (CAM) of the chicken embryo. Afterward, rhodamine B-labeled MSN-SP-PEPT were injected into the A549 tumor-bearing chicken embryos through a vein that was far from the tumor and allowed to accumulate in the tumoral mass for $48 \mathrm{~h}$ (Figure 4). The red fluorescence of the rhodamine B-labeled nanoparticles could be observed in the tumoral mass $48 \mathrm{~h}$ after their administration, confirming their ability to accumulate in the tumor via EPR effect (Figure 4A). Indeed, many marketed nanomedicines rely on this principle. ${ }^{66}$

The embryos were subsequently dissected and imaged (Figure 4B). Overall, MSN-SP-PEPT accumulated preferentially in the tumoral mass, and reduced accumulation was noted only in the kidneys and the liver, indicating the low clearance from the bloodstream. In addition, no significant organ damage was observed. These observations further confirmed the high colloidal stability of MSN-SP-PEPT, given that it could circulate for up to $72 \mathrm{~h}$ in the embryos without leading to aggregates, further emphasizing the biocompatibility of this drug delivery system.

Finally, MSN-SP-PEPT was loaded with DOX and a preliminary cytotoxicity study was performed. The drug-loaded nanoparticles were injected into the bloodstream of the A549 tumor-bearing chicken embryos through an injection in a vein, which were sacrificed after $72 \mathrm{~h}$. As observed in Figure 4C, the DOX-loaded nanocarrier could lessen the tumor weight by ca. $50 \%$ after only $72 \mathrm{~h}$. In this sense, it was found that at least 25 $\mu \mathrm{g}$ of free DOX were needed to obtain comparable results (data not shown), highlighting the suitability of MSN-SPPEPT to be employed as a nanomedicine in the treatment of tumors. 


\section{CONCLUSIONS}

In this research, redox-responsive mesoporous nanomaterials showing remarkable targeting and endosomal escape features was designed and produced. The particles were effective in controlling premature drug release thanks to the redoxresponsive self-immolative molecule. A simple and versatile molecule capable of targeting tumoral cells and to induce the escape from the endosomes was here synthesized for the first time and further grafted to the particles. The biological capabilities of this nanocarrier was extensively studied using 2D and 3D tumoral models, demonstrating remarkable cellular uptake and endosomal escape capabilities as well as excellent cytotoxic profile. In addition, the presence of HAVAB significantly enhanced the penetration of the particles in tumor spheroids. Finally, the particles were shown to passively accumulate in tumors of tumor-bearing chicken embryos. In addition, the here produced particles were shown to significantly reduce the weight of the tumor in a short space of time, confirming the potentiality of the engineered nanoparticle to addresses essential problems in current nanomedicine.

\section{MATERIALS AND METHODS}

The majority of the reagents employed in this work were bought from Sigma-Aldrich Inc.: ammonium nitrate; tetraethyl orthosilicate (TEOS); fluorescein isothiocyanate isomer I (FITC); cetyltrimethylammonium bromide (CTAB); 3-(aminopropyl)triethoxysilane (APTES); rhodamine B isothiocyanate (RhB); phenyl chloroformate; 4-sminobenzyl alcohol; fibutyltin dilaurate (DBTL); $N, N$-diisopropylethylamine (DIPEA); 2-mercaptoethanol; 2,2'-dipyridildisulfide; imidazole; 3-(triethoxysilyl)propyl isocyanate; $p$-toluenesulfonic acid (TsOH); tert-butyldimethylsilyl chloride (TBDMS-Cl); HS-PEG ${ }_{3500^{-}}$ $\mathrm{NH}_{2}$ (PEG-SH); Tris(2,2'-bipyridyl)dichlororuthenium(II) hexahydrate $(\mathrm{Ru})$; dithiothreitol (DTT); 2-chlorotrityl chloride resin; Fmoc5-Ava-OH (Ava); Fmoc-His(Trt)-OH (His); 1-hydroxybenzotriazole hydrate (HOBt); $N, N, N^{\prime}, N^{\prime}$-Tetramethyl-O-(1H-benzotriazol-1yl)uronium hexafluorophosphate (HBTU); dicyclohexylcarbodiimide (DCC); biotin $N$-hydroxysuccinimide ester (NHS-Biotin); triisopropylsilane (TIPS); trifluoroacetic acid (TFA); topotecan (TOP); doxorubicin (DOX); $N, N$-dimethylformamide (DMF); tetrahydrofuran (THF); dimethyl sulfoxide (DMSO); acetonitrile (AcN); dichloromethane (DCM). The rest of the chemicals (heptane, ethyl acetate, ethanol, etc.) were of the highest quality and employed as received.

Mesoporous Silica Nanoparticles. MSNs were produced following a modification of the Stöber method. ${ }^{67}$ Briefly, $1 \mathrm{~g}(2.74$ mmol) of CTAB, $480 \mathrm{~mL}$ of $\mathrm{H}_{2} \mathrm{O}$ and $3.5 \mathrm{~mL}$ of $\mathrm{NaOH}$ were mixed in a flask and heated to $80^{\circ} \mathrm{C}$. Then, $5 \mathrm{~mL}, 22.4 \mathrm{mmol}$, of TEOS were dropwisely added $(0.25 \mathrm{~mL} / \mathrm{min})$ over $20 \mathrm{~min}$, and then the reaction was subsequently stirred at $80{ }^{\circ} \mathrm{C}$ for a further $2 \mathrm{~h}$. Finally, the nanoparticles were collected by centrifugation and washed with $\mathrm{H}_{2} \mathrm{O}$ and ethanol. The organic template was removed by ionic exchange, dispersing the nanoparticles in $500 \mathrm{~mL}$ of an ethanolic solution (95\%) of $\mathrm{NH}_{4} \mathrm{NO}_{3}(10 \mathrm{mg} / \mathrm{mL})$ at $75{ }^{\circ} \mathrm{C}$ for $2 \mathrm{~h}$, and subsequently centrifuged and washed. The process was carried out three times. The nanoparticles were finally stored in absolute ethanol.

The cellular experiments were carried out using labeled MSNs. For that purpose, $1 \mathrm{mg}(0.002 \mathrm{mmol})$ of FITC or $1.07 \mathrm{mg}(0.002 \mathrm{mmol})$ of RhB were reacted with $2.2 \mu \mathrm{L}(0.009 \mathrm{mmol})$ of APTES in ethanol $(40 \mu \mathrm{L})$ for $2 \mathrm{~h}$. Then, this mixture was added to $5 \mathrm{~mL}(22.39 \mathrm{mmol})$ of TEOS, and the synthesis of MSNs was performed as abovedescribed.

The as-produced nanoparticles were characterized by fourier transformed infrared (FTIR) spectroscopy, X-ray diffraction (XRD), $\mathrm{N}_{2}$ adsorption analysis, thermogravimetric analysis (TGA analysis), dynamic light scattering (DLS) and zeta potential, and transmission electron microscopy (TEM).
Redox-Responsive Linker. Phenyl(4-(hydroxymethyl)phenyl)carbamate (1). Compound 1 was produced using a modified method published previously. ${ }^{68}$ In brief, $6 \mathrm{~g}(48.7 \mathrm{mmol})$ of 4-aminobenzyl alcohol was dissolved in $240 \mathrm{~mL}$ of THF/saturated aqueous sodium bicarbonate/water $(2: 2: 1)$. Then, $7 \mathrm{~mL}(55.8 \mathrm{mmol})$ of phenyl chloroformate was dropwisely added, and the reaction mixture was stirred at room temperature upon completion. Then, ethyl acetate was employed to extract compound $\mathbf{1}$, and the organic phase was washed several times with sodium bicarbonate, dried over sodium sulfate, and finally removed in vacuo. Finally, chloroform was used to recrystallize the crude, yielding compound 1 . The product was characterized by ${ }^{1} \mathrm{H}$ NMR.

Phenyl(4-((tert-butyldimethylsilyl)oxy)methyl)phenyl)carbamate (2). Compound 2 was synthesized using a published method with minor modifications. ${ }^{68}$ In brief, $1 \mathrm{~g}(4.2 \mathrm{mmol})$ of compound 1 and $0.31 \mathrm{~g}(4.52 \mathrm{mmol})$ of imidazole $(0.31 \mathrm{~g}, 4.52$ $\mathrm{mmol})$ were dissolved in $20 \mathrm{~mL}$ of DCM. Then, $0.75 \mathrm{~g}(5 \mathrm{mmol})$ of TBDMS-Cl were dropwisely added, and the reaction was stirred overnight at room temperature. Then, the imidazole salts generated were removed by filtration. Afterward, the solvent was removed in vacuo, and the crude was purified on a silica column (DCM), leading to compound 2 . The product was characterized by ${ }^{1} \mathrm{H}$ NMR

2-(Pyridin-2-yldisulfaneyl)ethan-1-ol (3). Compound 3 was produced following a published method. ${ }^{69}$ In brief, $2.42 \mathrm{~g}$ (10.98 $\mathrm{mmol})$ of $2,2^{\prime}$-dipyridyldisulfide and $0.26 \mathrm{~mL}(4.55 \mathrm{mmol})$ of acetic acid were dissolved in $40 \mathrm{~mL}$ of methanol. Then, $0.6 \mathrm{~mL}(8.52 \mathrm{mmol})$ of 2-mercaptoethanol in $10 \mathrm{~mL}$ of methanol was added dropwise, and the reaction was stirred for $48 \mathrm{~h}$ at room temperature. Afterward, the crude obtained after removing the solvent was purified on a silica column (heptane/ethyl acetate, 1:1) to yield compound 3 . The product was characterized by ${ }^{1} \mathrm{H}$ NMR.

2-(Pyridin-2-yldisulfaneyl)ethyl (4-(((tert-butyldimethylsilyl)oxy)methyl)phenyl)carbamate (4). First, $1.9 \mathrm{~g}(5.3 \mathrm{mmol})$ of compound 2 and $1.1 \mathrm{~g}(5.9 \mathrm{mmol})$ of compound 3 were dissolved in $40 \mathrm{~mL}$ of toluene. Then, $0.63 \mathrm{~mL}$ of DBTL were added, and the reaction was heated at $110{ }^{\circ} \mathrm{C}$ for $4 \mathrm{~h}$. Afterward, the solvent was evaporated to yield a crude that was purified on a silica column (heptane/ethyl acetate, $4: 1$ ), leading to compound 4 . The product was characterized by ${ }^{1} \mathrm{H}$ NMR.

2-(Pyridin-2-yldisulfaneyl)ethyl (4-(hydroxymethyl)phenyl)carbamate (5). First, $2 \mathrm{~g}$ (4.44 mmol) of compound 4 was dissolved in $50 \mathrm{~mL}$ of methanol and DCM (1:1). Then, $84.4 \mathrm{mg}$ of TsOH in 15 $\mathrm{mL}$ of methanol and DCM (1:1) was added, and the reaction was refluxed overnight. Afterward, the solvent was removed, and the crude was purified on a silica column (heptane/ethyl acetate, 1:2) to lead to compound 5. ${ }^{1} \mathrm{H}$ NMR was employed to characterize the final product.

MSNs Functionalized with the Redox-Responsive Linker (MSN-S). Initially, $148.5 \mu \mathrm{L}(0.6 \mathrm{mmol})$ of 3-(triethoxysilyl)propyl isocyanate was dissolved in $0.5 \mathrm{~mL}$ of dry DCM (vial 1). Separately, $0.24 \mathrm{~g}(0.72 \mathrm{mmol})$ of compound 5 was dissolved in $1.2 \mathrm{~mL}$ of dry DCM (vial 2). Afterward, vial 2 was dropwisely added dropwise over vial 1 , and the reaction was refluxed overnight. Once the silylation of the redox-responsive molecule was accomplished, the mixture was added to MSNs. For that purpose, $50 \mathrm{mg}$ of MSNs was dispersed in 5 $\mathrm{mL}$ of AcN. Then, silylated compound $\mathbf{5}$ was incorporated three times (additions spaced $2 \mathrm{~h}$ ), and the reaction was refluxed overnight. Then, the functionalized nanoparticles were collected by centrifugation and washed first with AcN and then with methanol to yield MSN-S. The nanoparticles were characterized in terms of FTIR spectroscopy, TGA, zeta potential, and DLS.

PEGylation of MSNs Functionalized with the RedoxResponsive Linker (MSN-SP). To PEGylate the as-produced redox-responsive particles, $45 \mathrm{mg}$ of MSN-S was dispersed in 15 $\mathrm{mL}$ of methanol. Then, $15 \mathrm{mg}(0.004 \mathrm{mmol})$ of PEG-SH in $1 \mathrm{~mL}$ of methanol was slowly incorporated into the mixture containing MSN$S$, and the reaction was stirred at room temperature overnight. Finally, the nanoparticles were collected by centrifugation and washed with methanol to yield MSN-SP, which was analyzed by FTIR spectroscopy, TGA, DLS and zeta potential, and TEM. 
Biotinylated Histidine-Rich Peptide (HAVAB). The biotinylated peptide (6, HAVAB, from Histidine-AminoValeric $\underline{\text { Acid-Biotin) }}$ was produced through solid-phase peptide synthesis, using Fmocprotected amino acids (Figure S3). Amide bonds were formed by adding $3.5 \mathrm{~mL}$ of DMF containing $300 \mathrm{mg}(0.792 \mathrm{mg})$ of HBTU, $107.06 \mathrm{mg}(0.792 \mathrm{mmol})$ of $\mathrm{HOBt}$, and $0.275 \mathrm{~mL}(1.58 \mathrm{mmol})$ of DIPEA to the resin $(200 \mathrm{mg}, 0.264 \mathrm{mmol})$. Then, the mixture was shaken overnight. After that, the resin was extensively rinsed with DMF to get rid of byproducts and unbounded amino acids. Then, 2 $\mathrm{mL}$ of piperidine (20\% in DMF) was added to cleave the Fmocprotecting group. Afterward, the resin was thoroughly rinsed with DMF, and the addition of the next amino acid was carried out as mentioned above. After incorporating the last amino acid, $270 \mathrm{mg}$ $(0.792 \mathrm{mmol})$ of NHS-Biotin and $0.275 \mathrm{~mL}(1.58 \mathrm{mmol})$ of DIPEA were dissolved in $6.5 \mathrm{~mL}$ of DMF, and the mixture was added to the resin and shaken in a $360^{\circ}$ vertical rotator overnight.

Once all the components of compound 6 were incorporated, the resin was thoroughly rinsed first with DMF and then with DCM. Then, $1.2 \mathrm{~mL}$ of a cleavage mixture (TFA (95\%), TIPS $(2.5 \%)$ and water $(2.5 \%)$ ) was added to the resin and kindly shaken for $2 \mathrm{~h}$, to release the peptide from the resin. Afterward, the solution was filtered and collected. Then, a small amount of cleavage mixture was used to rinse the resin, and both solutions were mixed. Such solution was precipitated into cold ether and centrifuged. Then, the crude was purified via molecular exclusion aschromatography, using Sephadex G-25 as stationary phase and water as mobile phase. Compound 6 was characterized by ${ }^{1} \mathrm{H}$ NMR and mass spectrometry.

Targeted Mesoporous Silica Nanoparticles (MSN-SP-PEPT). Thirty milligrams $(0.04 \mathrm{mmol})$ of compound $6,21.7 \mathrm{mg}(0.11 \mathrm{mmol})$ of DCC, and $12.1 \mathrm{mg}(0.11 \mathrm{mmol})$ of NHS were dissolved in $1.2 \mathrm{~mL}$ of dry DMF. The mixture was stirred for $1 \mathrm{~h}$ at room temperature for acid activation. Afterward, $35 \mathrm{mg}$ of MSN-SP were dispersed in 3.5 $\mathrm{mL}$ of dry DMF, and the solution that contained the acid-activated peptide was added dropwise. The reaction was stirred at room temperature for overnight. Afterward, the particles were centrifuged, washed with DMF, ethanol, water and methanol, leading to MSN-SPPEPT. The as-synthesized nanoparticles were characterized in terms of FTIR spectroscopy, TGA analysis, zeta potential, and DLS.

Release Experiments from MSN-SP. The response of the particles to redox stimuli was studied using DOX-loaded MSN-SP. For that purpose, MSN-SP were dispersed in a saturated solution of DOX in methanol and stirred overnight. Then, the particles were collected by centrifugation and washed until no drug could be observed in the supernatant.

The release experiment was performed in vial using Transwells (3 per condition). PBS $1 \times$ solutions in the presence or absence of DTT $(10 \mathrm{mM})$ were used to replicate the cytoplasm of cancer cells. Briefly, $10 \mathrm{mg}$ of particles were dispersed in $1.8 \mathrm{~mL}$ of the corresponding solution to then transfer $0.5 \mathrm{~mL}$ of the corresponding dispersion into each Transwell. The samples were kept with orbital stirring at $37{ }^{\circ} \mathrm{C}$ during the whole experiment. The media were removed every time point and studied using a fluorimeter $\left(\lambda_{\mathrm{exc}}=480 \mathrm{~nm}, \lambda_{\mathrm{em}}=600 \mathrm{~nm}\right)$. At every time point, old media was replaced by fresh media to keep the volume constant.

2D Cellular Experiments. Two-Dimensional Cell Cultures. The cellular experiments were carried out employing different cell lines (MC3T3-E1, A549, OVCAR8 and HeLa). A549 or HeLa cells $\left(20,000\right.$ cells $\left.\mathrm{cm}^{2}\right)$ were plated at $37^{\circ} \mathrm{C}$ in a humidified atmosphere of $\mathrm{CO}_{2}(5 \%)$ in DMEM $(1 \mathrm{~mL})$ containing FBS $(10 \%)$ and penicillinstreptomycin (1\%). OVCAR8 cells were plated analogously but in 1 $\mathrm{mL}$ of RPMI medium. MC3T3-E1 cells were plated similarly but employing $1 \mathrm{~mL}$ of $\alpha$-MEM. Afterward, different concentrations of the corresponding nanoparticles were incubated with the cells for different periods of time.

For the cytotoxicity studies, MSNs and MSN-SP-PEPT were dispersed in a saturated solution of DOX (for experiments with A549 cells) or TOP (for experiments with HeLa cells, see Supporting Information) in methanol, and the loading protocol was carried out as described in the previous section. For the endosomal escape experiments, MSNs and MSN-SP-PEPT were dispersed in a saturated solution of calcein (CAL) in methanol, and the loading protocol was carried out as described in the previous section.

Two-Dimensional Cell Viability. Cell viability was checked through the Alamar Blue method. For that purpose, the Alamar Blue solution ( $\mathrm{AbD}$ Serotec) at $10 \%(\mathrm{v} / \mathrm{v})$ was added to the cell culture at the studied time points, according to manufacturer protocols. After $4 \mathrm{~h}$, aliquots of each well $(200 \mu \mathrm{L})$ were placed in 96-well plates, and the absorbance intensity was recorded $\left(\lambda_{\text {exc }}=570\right.$ $\mathrm{nm} ; \lambda_{\mathrm{em}}=600 \mathrm{~nm}$ ) using a spectrophotometer (Unicam UV-500 UVvisible).

$2 D$ Internalization Studies. Cells were seeded following the abovedescribed protocol for the fluorescence microscopy studies. Rhodamine B-labeled nanoparticles were employed.

MC3T3-E1 or HeLa cells were seeded in each well of a 6-well plate for the flow cytometry studies. After $24 \mathrm{~h}$, different concentrations of fluorescein-labeled nanoparticles were added to the cells. After $2 \mathrm{~h}$, cells were washed with PBS and incubated with trypsin-EDTA at 37 ${ }^{\circ} \mathrm{C}$ for cell detachment. After 5 min, culture medium was added, and the cells were collected by centrifugation $(10 \mathrm{~min}, 1500 \mathrm{rpm})$. The cells were resuspended in fresh medium and the fluorescence from the surface of the cells was quenched using trypan blue $(0.4 \%)$ to avoid the detection of fluorescence from noninternalized particles. Flow cytometry was carried out at an excitation wavelength of $488 \mathrm{~nm}$ and measured at $530 \mathrm{~nm}$. The trigger was set for the green fluorescence channel. The data acquisition and analysis conditions were established using positive and negative controls with the CellQuest Program of Becton-Dickinson. The conditions were kept constant during all the experiments. Three replicas per condition were employed. At least 10000 cells of each sample were analyzed for statistical significance.

OVCAR8 or A549 cells were placed with different concentrations of rhodamine B-labeled nanoparticles (MSNs or MSN-SP-PEPT) for confocal laser scanning microscopy. After $2 \mathrm{~h}$, noninternalized nanoparticles were removed by rinsing each well with iced PBS. The cells were fixed with cold ethanol (75\%). Then, ethanol was removed, and PBS was employed to rinse the wells. The cell nuclei were stained using DAPI, and then the wells were washed with iced PBS. The internalization was determined by confocal laser scanning microscopy $\left(\lambda_{\text {exc }}=488 \mathrm{~nm} ; \lambda_{\text {em }}=610 \mathrm{~nm}\right)$.

3D Cellular Experiments. Three-Dimensional Cell Cultures. Spheroids were grown in a 96 U-shaped-well plate. To that aim, OVCAR8 or A549 cells ( 10000 cells) were added to a well containing $200 \mu \mathrm{L}$ of either DMEM or RPMI medium, respectively. Cellular spheroids were grown for a week in a humidified $\mathrm{CO}_{2}$ atmosphere at $37^{\circ} \mathrm{C}$.

Spheroid Viability. The effect of the particles on the cellular viability was assessed using A549 spheroids. Different concentrations of DOX-loaded and drug-free nanoparticles were incubated with the spheroids for $24 \mathrm{~h}$. After that, noninternalized nanoparticles were removed by rinsing the wells with PBS, and $200 \mu \mathrm{L}$ of the appropriate culture medium were added. The spheroids were then maintained for a week in a humidified atmosphere at $37^{\circ} \mathrm{C}$ and imaged at different time points. The viability of the spheroids was studied through the variation in the spheroid volume, using the formula: $:^{63,70}$

$$
V=0.5 *(\operatorname{short} \phi)^{2} * \operatorname{long} \phi
$$

where " $V$ " indicates the volume of the spheroid and "short $\phi$ " and "long $\phi$ " indicate the short and long diameters of the spheroid, respectively. The values for each diameter were determined analyzing each image with the software ImageJ.

Internalization Studies in Spheroids. The studies of internalization were performed employing OVCAR8 or A549 tumor spheroids. For that purpose, rhodamine B-labeled nanoparticles (MSNs or MSN-SP-PEPT) were incubated with the cellular spheroids for $24 \mathrm{~h}$. Then, the wells were washed with PBS $1 \times$ to removed non internalized nanoparticles and the samples were transferred to an Eppendorf tube. A paraformaldehyde solution (4\%) at $4{ }^{\circ} \mathrm{C}$ was employed to fix the spheroids overnight. After that, the spheroids were rinsed with iced PBS $1 \times$ and treated with cold methanol for 0.5 h. Finally, the samples were kept in PBS $1 \times$ in the fridge for confocal 
microscopy. The confocal microscope generated multiple twodimensional images along the $z$-axis, from the top to the bottom, that were then stacked using the software ImageJ to accomplish the $2 \mathrm{D}$ projection of the $3 \mathrm{D}$ spheroids.

Chicken Embryo Experiments. Tumor Formation. Freshly fertilized chicken eggs were kept for 10 days in a humidified atmosphere $(60 \%)$ at $37{ }^{\circ} \mathrm{C}$. On day 10 , a small portion of the eggshell was cut out, and a Teflon ring was then placed onto the CAM membrane. After that, 100000 A549 cells were carefully left in the ring, and the window was closed to grow the tumor for 3 days.

Biodistribution Studies. Once the tumoral mass was completely developed, a small window was opened in a different place, and rhodamine B-labeled MSN-SP-PEPT were administered, using a syringe, in a vein which was far from the tumor. Then, the window was closed again and the embryos were kept in the incubator 2 more days. Afterward, the eggs were visualized using a fluorescence stereomicroscope and finally dissected to assess the distribution of the particles in the tumor and the different organs.

Tumor Reduction. DOX-loaded rhodamine B-labeled MSN-SPPEPT were administered following the protocol described above at a concentration of $100 \mu \mathrm{g} / \mathrm{mL}$. The embryos $(n=5)$ were incubated for $72 \mathrm{~h}$ and subsequently dissected to measure the weight of the tumors. A control group without nanoparticles $(n=5)$ was also included.

\section{ASSOCIATED CONTENT}

\section{s) Supporting Information}

The Supporting Information is available free of charge at https://pubs.acs.org/doi/10.1021/acsami.0c21507.

Summary of the materials produced in this work; Characterization of MSNs and functionalized MSNs (TGA analysis, XRD, FTIR, $\mathrm{N}_{2}$ adsorption-desorption isotherms, DLS measurements and zeta potential); Schematic representation of the synthesis of the redoxresponsive linker with ${ }^{1} \mathrm{H}$ NMR characterization; Optimization of the grafting protocol $\left({ }^{1} \mathrm{H}\right.$ NMR, TGA and FTIR); Validation of the redox-responsiveness (elemental analysis, FTIR); Additional release experiment; Schematic synthesis of HAVAB with ${ }^{1} \mathrm{H}$ NMR and mass spectrometry characterization; Effect of biotin on $2 \mathrm{D}$ internalization (flow cytometry and confocal microscopy); Endolysosomal escape using LysoTracker; Additional 2D cytotoxicity; 3D cellular uptake in OVCAR8 spheroids and depth penetration; Additional cytotoxicity in A549 spheroids; Schematic representation of the chicken embryo model (PDF)

\section{AUTHOR INFORMATION}

\section{Corresponding Authors}

Fuyuhiko Tamanoi - Institute for Integrated Cell-Material Sciences, Institute for Advanced Study, Kyoto University, Kyoto 606-8501, Japan; Department of Microbiology, Immunology and Molecular Genetics, University of California, Los Angeles, California 90095, United States; (1) orcid.org/0000-0002-4220-6408;

Email: tamanoi.fuyuhiko.2c@kyoto-u.ac.jp

María Vallet-Regí - Chemistry in Pharmaceutical Sciences, School of Pharmacy, Universidad Complutense de Madrid, Madrid 28040, Spain; Networking Research Center on Bioengineering, Biomaterials and Nanomedicine (CIBERBBN), Madrid 28029, Spain; (1) orcid.org/0000-00026104-4889; Email: vallet@ucm.es

\section{Authors}

Miguel Gisbert-Garzarán - Chemistry in Pharmaceutical Sciences, School of Pharmacy, Universidad Complutense de Madrid, Madrid 28040, Spain; Networking Research Center on Bioengineering, Biomaterials and Nanomedicine (CIBER$B B N)$, Madrid 28029, Spain; (1) orcid.org/0000-00019815-0354

Daniel Lozano - Chemistry in Pharmaceutical Sciences, School of Pharmacy, Universidad Complutense de Madrid, Madrid 28040, Spain; Networking Research Center on Bioengineering, Biomaterials and Nanomedicine (CIBER$B B N)$, Madrid 28029, Spain; 이이.org/0000-00015902-9201

Kotaro Matsumoto - Institute for Integrated Cell-Material Sciences, Institute for Advanced Study, Kyoto University, Kyoto 606-8501, Japan; orcid.org/0000-0002-49270103

Aoi Komatsu - Institute for Integrated Cell-Material Sciences, Institute for Advanced Study, Kyoto University, Kyoto 6068501, Japan; 이이.orid.0000-0003-2322-8692

Miguel Manzano - Chemistry in Pharmaceutical Sciences, School of Pharmacy, Universidad Complutense de Madrid, Madrid 28040, Spain; Networking Research Center on Bioengineering, Biomaterials and Nanomedicine (CIBER$B B N)$, Madrid 28029, Spain; ㅇo orcid.org/0000-00016238-6111

Complete contact information is available at: https://pubs.acs.org/10.1021/acsami.0c21507

\section{Author Contributions}

The manuscript was written through contributions of all authors. All authors have given approval to the final version of the manuscript.

\section{Funding}

This project received funding from the following grants: ERC2015 AdG (VERDI), Proposal No. 694160; Japan Society for the Promotion of Science (JSPS) Grants-in-Aid for Scientific Research (KAKENHI), Grant No. JP15K21764 and Japan Agency for Medical Research and Development (AMED) Grant No. 19ck0106469h0001.

\section{Notes}

The authors declare no competing financial interest.

\section{ACKNOWLEDGMENTS}

The authors would like to acknowledge funding from the following grants: ERC-2015 AdG (VERDI), Proposal No. 694160; Japan Society for the Promotion of Science (JSPS) Grants-in-Aid for Scientific Research (KAKENHI), Grant No. JP15K21764 and Japan Agency for Medical Research and Development (AMED) Grant No. 19ck0106469h0001.

\section{REFERENCES}

(1) Bray, F.; Ferlay, J.; Soerjomataram, I.; Siegel, R. L.; Torre, L. A.; Jemal, A. Global Cancer Statistics 2018: GLOBOCAN Estimates of Incidence and Mortality Worldwide for 36 Cancers in 185 Countries. Ca-Cancer J. Clin. 2018, 68, 394-424.

(2) Stewart, B.; Wild, C. World Cancer Report; World Health Organization Press: Lyon, 2014.

(3) Shi, J.; Kantoff, P. W.; Wooster, R.; Farokhzad, O. C. Cancer Nanomedicine: Progress, Challenges and Opportunities. Nat. Rev. Cancer 2017, 17, 20-37. 
(4) Wicki, A.; Witzigmann, D.; Balasubramanian, V.; Huwyler, J. Nanomedicine in Cancer Therapy: Challenges, Opportunities, and Clinical Applications. J. Controlled Release 2015, 200, 138-157.

(5) Rosenblum, D.; Joshi, N.; Tao, W.; Karp, J. M.; Peer, D. Progress and Challenges towards Targeted Delivery of Cancer Therapeutics. Nat. Commun. 2018, 9, No. 1410.

(6) Vivero-Escoto, J. L.; Slowing, I. I.; Trewyn, B. G.; Lin, V. S.-Y. Mesoporous Silica Nanoparticles for Intracellular Controlled Drug Delivery. Small 2010, 6, 1952-1967.

(7) Lu, J.; Liong, M.; Li, Z.; Zink, J. I.; Tamanoi, F. Biocompatibility, Biodistribution, and Drug-Delivery Efficiency of Mesoporous Silica Nanoparticles for Cancer Therapy in Animals. Small 2010, 6, 17941805.

(8) Manzano, M.; Vallet-Regí, M. Mesoporous Silica Nanoparticles for Drug Delivery. Adv. Funct. Mater. 2020, 30, No. 1902634.

(9) Vallet-Regi, M.; Ramila, A.; del Real, R. P.; Perez-Pariente, J. A New Property of MCM-41: Drug Delivery System. Chem. Mater. 2001, 13, 308-311.

(10) Gisbert-Garzarán, M.; Manzano, M.; Vallet-Regí, M. Mesoporous Silica Nanoparticles for the Treatment of Complex Bone Diseases: Bone Cancer, Bone Infection and Osteoporosis. Pharmaceutics 2020, 12, No. 83.

(11) Moreira, A. F.; Dias, D. R.; Correia, I. J. Stimuli-Responsive Mesoporous Silica Nanoparticles for Cancer Therapy: A Review. Microporous Mesoporous Mater. 2016, 236, 141-157.

(12) Gisbert-Garzarán, M.; Manzano, M.; Vallet-Regí, M. pHResponsive Mesoporous Silica and Carbon Nanoparticles for Drug Delivery. Bioengineering 2017, 4, No. 3.

(13) Argyo, C.; Weiss, V.; Brauchle, C.; Bein, T. Multifunctional Mesoporous Silica Nanoparticles as a Universal Platform for Drug Delivery. Chem. Mater. 2014, 26, 435-451.

(14) Colilla, M.; González, B.; Vallet-Regí, M. Mesoporous Silica Nanoparticles for the Design of Smart Delivery Nanodevices. Biomater. Sci. 2013, 1, 114-134.

(15) Gisbert-Garzarán, M.; Lozano, D.; Vallet-Regí, M.; Manzano, M. Self-Immolative Polymers as Novel PH-Responsive Gate Keepers for Drug Delivery. RSC Adv. 2017, 7, 132-136.

(16) Juárez, L. A.; Añón, E.; Giménez, C.; Sancenón, F.; MartínezMáñez, R.; Costero, A. M.; Gaviña, P.; Parra, M.; Bernardos, A. SelfImmolative Linkers as Caps for the Design of Gated Silica Mesoporous Supports. Chem. - Eur. J. 2016, 22, 14126-14130.

(17) Gisbert-Garzarán, M.; Berkmann, J. C.; Giasafaki, D.; Lozano, D.; Spyrou, K.; Manzano, M.; Steriotis, T.; Duda, G. N.; SchmidtBleek, K.; Charalambopoulou, G.; Vallet-Regí, M. Engineered PHResponsive Mesoporous Carbon Nanoparticles for Drug Delivery. ACS Appl. Mater. Interfaces 2020, 12, 14946-14957.

(18) Gisbert-Garzarán, M.; Manzano, M.; Vallet-Regí, M. SelfImmolative Chemistry in Nanomedicine. Chem. Eng. J. 2018, 340, 24-31.

(19) Blencowe, C. A.; Russell, A. T.; Greco, F.; Hayes, W.; Thornthwaite, D. W. Self-Immolative Linkers in Polymeric Delivery Systems. Polym. Chem. 2011, 2, 773-790.

(20) Danial, M.; Telwatte, S.; Tyssen, D.; Cosson, S.; Tachedjian, G.; Moad, G.; Postma, A. Combination Anti-HIV Therapy via Tandem Release of Prodrugs from Macromolecular Carriers. Polym. Chem. 2016, 7, 7477-7487.

(21) Latorre, A.; Posch, C.; Garcimartin, Y.; Celli, A.; Sanlorenzo, M.; Vujic, I.; Ma, J.; Zekhtser, M.; Rappersberger, K.; Ortiz-Urda, S.; Somoza, A. DNA and Aptamer Stabilized Gold Nanoparticles for Targeted Delivery of Anticancer Therapeutics. Nanoscale 2014, 6, $7436-7442$

(22) Peng, Z.; Xie, Y.; Wang, Y.; Li, J.; Oupicky, D. Dual-Function Polymeric HPMA Prodrugs for the Delivery of MiRNA. Mol. Pharmaceutics 2017, 14, 1395-1404.

(23) Xie, Y.; Murray-stewart, T.; Wang, Y.; Yu, F.; Li, J.; Marton, L. J.; Casero, R. A.; Oupický, D. Self-Immolative Nanoparticles for Simultaneous Delivery of MicroRNA and Targeting of Polyamine Metabolism in Combination Cancer Therapy. J. Controlled Release 2017, 246, 110-119.
(24) Tan, X.; Lu, X.; Jia, F.; Liu, X.; Sun, Y.; Logan, J. K.; Zhang, K. Blurring the Role of Oligonucleotides: Spherical Nucleic Acids as a Drug Delivery Vehicle. J. Am. Chem. Soc. 2016, 138, 10834-10837.

(25) Liu, G.; Wang, X.; Hu, J.; Zhang, G.; Liu, S. Self-Immolative Polymersomes for High-Efficiency Triggered Release and Programmed Enzymatic Reactions. J. Am. Chem. Soc. 2014, 136, 74927497.

(26) Blanco, E.; Shen, H.; Ferrari, M. Principles of Nanoparticle Design for Overcoming Biological Barriers to Drug Delivery. Nat. Biotechnol. 2015, 33, 941-951.

(27) Gisbert-Garzarán, M.; Vallet-Regí, M. Influence of the Surface Functionalization on the Fate and Performance of Mesoporous Silica Nanoparticles. Nanomaterials 2020, 10, No. 916.

(28) Maeda, H.; Nakamura, H.; Fang, J. The EPR Effect for Macromolecular Drug Delivery to Solid Tumors: Improvement of Tumor Uptake, Lowering of Systemic Toxicity, and Distinct Tumor Imaging in Vivo. Adv. Drug Delivery Rev. 2013, 65, 71-79.

(29) Foroozandeh, P.; Aziz, A. A. Insight into Cellular Uptake and Intracellular Trafficking of Nanoparticles. Nanoscale Res. Lett. 2018, 13, 339.

(30) Baeza, A.; Colilla, M.; Vallet-Regí, M. Advances in Mesoporous Silica Nanoparticles for Targeted Stimuli-Responsive Drug Delivery. Expert Opin. Drug Delivery 2015, 12, 319-337.

(31) Niedermayer, S.; Weiss, V.; Herrmann, A.; Schmidt, A.; Datz, S.; Müller, K.; Wagner, E.; Bein, T.; Bräuchle, C. Multifunctional Polymer-Capped Mesoporous Silica Nanoparticles for PH-Responsive Targeted Drug Delivery. Nanoscale 2015, 7, 7953-7964.

(32) Traverso, N.; Ricciarelli, R.; Nitti, M.; Marengo, B.; Furfaro, A. L.; Pronzato, M. A.; Marinari, U. M.; Domenicotti, C. Role of Glutathione in Cancer Progression and Chemoresistance. Oxid. Med. Cell. Longev. 2013, No. 972913.

(33) Guo, X.; Cheng, Y.; Zhao, X.; Luo, Y.; Chen, J.; Yuan, W. E. Advances in Redox-Responsive Drug Delivery Systems of Tumor Microenvironment. J. Nanobiotechnol. 2018, 16, 74-83.

(34) Sagi, A.; Weinstain, R.; Karton, N.; Shabat, D. Self-Immolative Polymers. J. Am. Chem. Soc. 2008, 130, 5434-5435.

(35) Iamsaard, S.; Seidi, F.; Dararatana, N.; Crespy, D. RedoxResponsive Polymer with Self-Immolative Linkers for the Release of Payloads. Macromol. Rapid Commun. 2018, 39, No. 1800071.

(36) Liu, B.; Ejaz, W.; Gong, S.; Kurbanov, M.; Canakci, M.; Anson, F.; Thayumanavan, S. Engineered Interactions with Mesoporous Silica Facilitate Intracellular Delivery of Proteins and Gene Editing. Nano Lett. 2020, 20, 4014-4021.

(37) Paris, J. L.; Villaverde, G.; Cabañas, M. V.; Manzano, M.; Vallet-Regí, M. From Proof-of-Concept Material to PEGylated and Modularly Targeted Ultrasound-Responsive Mesoporous Silica Nanoparticles. J. Mater. Chem. B 2018, 6, 2785-2794.

(38) Wani, A.; Savithra, G. H. L.; Abyad, A.; Kanvinde, S.; Li, J.; Brock, S.; Oupický, D. Surface PEGylation of Mesoporous Silica Nanorods (MSNR): Effect on Loading, Release, and Delivery of Mitoxantrone in Hypoxic Cancer Cells. Sci. Rep. 2017, 7, 2274.

(39) Ren, W. X.; Han, J.; Uhm, S.; Jang, Y. J.; Kang, C.; Kim, J. H.; Kim, J. S. Recent Development of Biotin Conjugation in Biological Imaging, Sensing, and Target Delivery. Chem. Commun. 2015, 51, 10403-10418.

(40) Lv, G.; Qiu, L.; Liu, G.; Wang, W.; Li, K.; Zhao, X.; Lin, J. PH Sensitive Chitosan-Mesoporous Silica Nanoparticles for Targeted Delivery of a Ruthenium Complex with Enhanced Anticancer Effects. Dalt. Trans. 2016, 45, 18147-18155.

(41) Li, Z.; Zhang, Y.; Zhang, K.; Wu, Z.; Feng, N. BiotinylatedLipid Bilayer Coated Mesoporous Silica Nanoparticles for Improving the Bioavailability and Anti-Leukaemia Activity of Tanshinone IIA. Artif. Cells, Nanomed., Biotechnol. 2018, 46, 578-587.

(42) Lv, G.; Li, K.; Qiu, L.; Peng, Y.; Zhao, X.; Li, X.; Liu, Q.; Wang, S.; Lin, J. Enhanced Tumor Diagnostic and Therapeutic Effect of Mesoporous Silica Nanoparticle-Mediated Pre-Targeted Strategy. Pharm. Res. 2018, 35, No. 63. 
(43) Li, S.; Hong, M. Protonation, Tautomerization, and Rotameric Structure of Histidine: A Comprehensive Study by Magic-AngleSpinning Solid-State NMR. J. Am. Chem. Soc. 2011, 133, 1534-1544.

(44) Hama, S.; Itakura, S.; Nakai, M.; Nakayama, K.; Morimoto, S.; Suzuki, S.; Kogure, K. Overcoming the Polyethylene Glycol Dilemma via Pathological Environment-Sensitive Change of the Surface Property of Nanoparticles for Cellular Entry. J. Controlled Release 2015, 206, 67-74.

(45) Wang, C. Y.; Huang, L. Polyhistidine Mediates an AcidDependent Fusion of Negatively Charged Liposomes. Biochemistry 1984, 23, 4409-4416.

(46) Buré, C.; Maget, R.; Delmas, A. F.; Pichon, C.; Midoux, P. Histidine-Rich Peptide: Evidence for a Single Zinc-Binding Site on H5WYG Peptide That Promotes Membrane Fusion at Neutral PH. J. Mass Spectrom. 2009, 44, 81-89.

(47) Krishnan, A.; Verma, S. K.; Mani, P.; Gupta, R.; Kundu, S.; Sarkar, D. P. A Histidine Switch in Hemagglutinin-Neuraminidase Triggers Paramyxovirus-Cell Membrane Fusion. J. Virol. 2009, 83, $1727-1741$

(48) Field, L. D.; Delehanty, J. B.; Chen, Y.; Medintz, I. L. Peptides for Specifically Targeting Nanoparticles to Cellular Organelles: Quo Vadis? Acc. Chem. Res. 2015, 48, 1380-1390.

(49) Sun, P.; Huang, W.; Kang, L.; Jin, M.; Fan, B.; Jin, H.; Wang, Q.-m.; Gao, Z. SiRNA-Loaded Poly(Histidine-Arginine)6-Modificed Chitosan Nanoparticle With Enhanced Cell- Penetrating and Endosomal Escape Capacities for Suppressing Breast Tumor Metastasis. Int. J. Nanomed. 2017, 12, 3221-3234.

(50) Midoux, P.; Pichon, C.; Yaouanc, J. J.; Jaffrès, P. A. Chemical Vectors for Gene Delivery: A Current Review on Polymers, Peptides and Lipids Containing Histidine or Imidazole as Nucleic Acids Carriers. Br. J. Pharmacol. 2009, 157, 166-178.

(51) Liang, K.; Richardson, J. J.; Ejima, H.; Such, G. K.; Cui, J.; Caruso, F. Peptide-Tunable Drug Cytotoxicity via One-Step Assembled Polymer Nanoparticles. Adv. Mater. 2014, 26, 2398-2402.

(52) Selby, L. I.; Cortez-Jugo, C. M.; Such, G. K.; Johnston, A. P. R. Nanoescapology: Progress toward Understanding the Endosomal Escape of Polymeric Nanoparticles. Wiley Interdiscip. Rev. Nanomedicine Nanobiotechnology 2017, 9, No. e1452.

(53) Simões, S.; Slepushkin, V.; Düzgünes, N.; Pedroso de Lima, M. C. On the Mechanisms of Internalization and Intracellular Delivery Mediated by PH-Sensitive Liposomes. Biochim. Biophys. Acta, Biomembr. 2001, 1515, 23-37.

(54) Kono, K.; Igawa, T.; Takagishi, T. Cytoplasmic Delivery of Calcein Mediated by Liposomes Modified with a PH-Sensitive Poly(Ethylene Glycol) Derivative. Biochim. Biophys. Acta, Biomembr. 1997, 1325, 143-154.

(55) Lattin, J. R.; Javadi, M.; McRae, M.; Pitt, W. G. Cytosolic Delivery via Escape from the Endosome Using Emulsion Droplets and Ultrasound. J. Drug Target. 2015, 23, 469-479.

(56) Smith, S. A.; Selby, L. I.; Johnston, A. P. R.; Such, G. K. The Endosomal Escape of Nanoparticles: Toward More Efficient Cellular Delivery. Bioconjugate Chem. 2019, 30, 263-272.

(57) Sun, X.; Luo, Y.; Huang, L.; Yu, B. Y.; Tian, J. A PeptideDecorated and Curcumin-Loaded Mesoporous Silica Nanomedicine for Effectively Overcoming Multidrug Resistance in Cancer Cells. RSC Adv. 2017, 7, 16401-16409.

(58) Zanoni, M.; Piccinini, F.; Arienti, C.; Zamagni, A.; Santi, S.; Polico, R.; Bevilacqua, A.; Tesei, A. 3D Tumor Spheroid Models for in Vitro Therapeutic Screening: A Systematic Approach to Enhance the Biological Relevance of Data Obtained. Sci. Rep. 2016, 6, No. 19103.

(59) Ye, D.; Raghnaill, M. N.; Bramini, M.; Mahon, E.; Åberg, C.; Salvati, A.; Dawson, K. A. Nanoparticle Accumulation and Transcytosis in Brain Endothelial Cell Layers. Nanoscale 2013, 5, 1115311165.

(60) Kim, J. A.; Aberg, C.; Salvati, A.; Dawson, K. A. Role of Cell Cycle on the Cellular Uptake and Dilution of Nanoparticles in a Cell Population. Nat. Nanotechnol. 2012, 7, 62-68.
(61) Lu, H.; Utama, R. H.; Kitiyotsawat, U.; Babiuch, K.; Jiang, Y.; Stenzel, M. H. Enhanced Transcellular Penetration and Drug Delivery by Crosslinked Polymeric Micelles into Pancreatic Multicellular Tumor Spheroids. Biomater. Sci. 2015, 3, 1085-1095.

(62) Cui, Y.; Shan, W.; Zhou, R.; Liu, M.; Wu, L.; Guo, Q.; Zheng, Y.; Wu, J.; Huang, Y. The Combination of Endolysosomal Escape and Basolateral Stimulation to Overcome the Difficulties of "Easy Uptake Hard Transcytosis" of Ligand-Modified Nanoparticles in Oral Drug Delivery. Nanoscale 2018, 10, 1494-1507.

(63) Lee, J. Y.; Chung, S. J.; Cho, H. J.; Kim, D. D. Phenylboronic Acid-Decorated Chondroitin Sulfate A-Based Theranostic Nanoparticles for Enhanced Tumor Targeting and Penetration. Adv. Funct. Mater. 2015, 25, 3705-3717.

(64) Vu, B. T.; Shahin, S. A.; Croissant, J.; Fatieiev, Y.; Matsumoto, K.; Le-Hoang Doan, T.; Yik, T.; Simargi, S.; Conteras, A.; Ratliff, L.; Jimenez, C. M.; Raehm, L.; Khashab, N.; Durand, J.-O.; Glackin, C.; Tamanoi, F. Chick Chorioallantoic Membrane Assay as an in Vivo Model to Study the Effect of Nanoparticle-Based Anticancer Drugs in Ovarian Cancer. Sci. Rep. 2018, 8, 8524.

(65) Komatsu, A.; Matsumoto, K.; Saito, T.; Muto, M.; Tamanoi, F. Patient Derived Chicken Egg Tumor Model (PDcE Model): Current Status and Critical Issues. Cells 2019, 8, No. 440.

(66) Grodzinski, P.; Kircher, M.; Goldberg, M.; Gabizon, A. Integrating Nanotechnology into Cancer Care. ACS Nano 2019, 13, $7370-7376$

(67) Baeza, A.; Guisasola, E.; Torres-Pardo, A.; González-Calbet, J. M.; Melen, G. J.; Ramírez, M.; Vallet-Regí, M. Hybrid EnzymePolymeric Capsules/Mesoporous Silica Nanodevice for in Situ Cytotoxic Agent Generation. Adv. Funct. Mater. 2014, 24, 46254633.

(68) Robbins, J. S.; Schmid, K. M.; Phillips, S. T. Effects of Electronics, Aromaticity, and Solvent Polarity on the Rate of Azaquinone-Methide-Mediated Depolymerization of Aromatic Carbamate Oligomers. J. Org. Chem. 2013, 78, 3159-3169.

(69) Horikawa, R.; Sunayama, H.; Kitayama, Y.; Takano, E.; Takeuchi, T. A Programmable Signaling Molecular Recognition Nanocavity Prepared by Molecular Imprinting and Post-Imprinting Modifications. Angew. Chem. 2016, 128, 13217-13221.

(70) Mai, N. X. D.; Birault, A.; Matsumoto, K.; Ta, H. K. T.; Intasaard, S. G.; Morrison, K.; Thang, P. B.; Doan, T. L. H.; Tamanoi, F. Biodegradable Periodic Mesoporous Organosilica (BPMO) Loaded with Daunorubicin: A Promising Nanoparticle-Based Anticancer Drug. ChemMedChem 2020, 15, 593-599. 